\title{
Measuring the Leptonic CP Phase in Neutrino Oscillations with Non-Unitary Mixing
}

\author{
Pedro Pasquini* \\ AHEP Group, Institut de Física Corpuscular - C.S.I.C./Universitat de València, Parc Cientific \\ de Paterna. C/Catedratico José Beltrán, 2 E-46980 Paterna (València) - SPAIN \\ Instituto de Física Gleb Wataghin - UNICAMP, 13083-859, Campinas SP, Brazil
}

E-mail: pasquini@ifi.unicamp.br

\section{Shao-Feng Ge}

Max-Planck-Institut für Kernphysik, Heidelberg 69117, Germany

E-mail: gesf02@gmail.com

\section{Tórtola}

AHEP Group, Institut de Física Corpuscular - C.S.I.C./Universitat de València, Parc Cientific de Paterna. C/Catedratico José Beltrán, 2 E-46980 Paterna (València) - SPAIN

E-mail: mariameific.uv.es

\section{J. W. F. Valle}

AHEP Group, Institut de Física Corpuscular - C.S.I.C./Universitat de València, Parc Cientific de Paterna. C/Catedratico José Beltrán, 2 E-46980 Paterna (València) - SPAIN

E-mail: valledific.uv.es, URL: http://astroparticles.es/

\begin{abstract}
Non-unitary neutrino mixing implies an extra CP violating phase that can fake the leptonic Dirac $\mathrm{CP}$ phase $\delta_{C P}$ of the simplest three-neutrino mixing benchmark scheme. This would hinder the possibility of probing for $\mathrm{CP}$ violation in accelerator-type experiments. We take T2K and T2HK as examples to demonstrate the degeneracy between the "standard" (or "non-unitary") and "nonunitary" CP phases. We find, under the assumption of non-unitary mixing, that their CP sensitivities severely deteriorate. Fortunately, the Tokai'N Toyama to Kamioka (TNT2K) proposal of supplementing $\mathrm{T} 2(\mathrm{H}) \mathrm{K}$ with a $\mu \mathrm{DAR}$ source for better measurement of $\delta_{C P}$ can partially break the $\mathrm{CP}$ degeneracy by probing both $\cos \delta_{C P}$ and $\sin \delta_{C P}$ dependences in the wide spectrum of the $\mu$ DAR flux. We also show that the further addition of a near detector to the $\mu$ DAR setup can eliminate the degeneracy completely. This summary is based on ref [1].
\end{abstract}

Neutrino Oscillation Workshop

4 - 11 September, 2016

Otranto (Lecce, Italy)

\footnotetext{
* Speaker.
} 


\section{Introduction}

If neutrinos acquire mass from the general seesaw mechanism through the exchange of $\mathrm{SU}(3)_{\mathrm{c}} \otimes \mathrm{SU}(2)_{\mathrm{L}} \otimes \mathrm{U}(1)_{\mathrm{Y}}$ singlet heavy messenger fermions, these extra neutrino states mix with the standard $v_{e}, v_{\mu}, v_{\tau}$, and then the neutrino mixing needs to be extended to go beyond $3 \times 3$, However if the extra neutrinos are heavy they will not be accessible to oscillations. In such case, the original $3 \times 3$ unitary mixing $U$ is replaced by a truncated non-unitary mixing matrix $N$ which will effectively describe neutrino propagation. This can be written as $[2,3]$

$$
N=N^{N P} U=\left(\begin{array}{ccc}
\alpha_{11} & 0 & 0 \\
\alpha_{21} & \alpha_{22} & 0 \\
\alpha_{31} & \alpha_{32} & \alpha_{33}
\end{array}\right) U
$$

Current experiments, mainly involving electron and muon neutrinos, are sensitive to three of these parameters: $\alpha_{11}, \alpha_{22}$ and $\alpha_{21}$. Note that the latter is complex and therefore we end up with three additional real parameters and one new complex phase $\phi \equiv-\arg \left(\alpha_{21}\right)$.

There are a number of constraints on non-unitarity, such as those that follow from weak universality considerations. These include both universality as well as oscillation limits. The main emphasis of our paper will be on experiments providing robust model-independent bounds on nonunitarity relying only on neutrino processes. For this reason here we will concentrate on the following bound on $\alpha_{21}$ due the non-observation of $v_{\mu}$ to $v_{e}$ conversion at the NOMAD experiment, only relevant neutrino oscillation experiment. We implement this bound as prior in the NuPro package [4] as

$$
\left[\sin ^{2}\left(2 \theta_{\mu e}\right)\right]_{e f f}=2\left|\alpha_{21}\right|^{2} \leq 0.0014 \quad \text { @ 90\%C.L. }
$$

We will show how non-unitary mixing can deteriorate the $\mathrm{CP}$ measurement in neutrino oscillation experiments under the current model-independent constraints. What we propose in this paper can improve not only the constraint on non-unitary mixing but also the resulting CP sensitivity [5].

\section{Faking the Dirac CP Phase with Non-Unitarity}

In order to study to what extent the standard CP phase $\delta_{C P}$ can be faked by the non-unitary $\mathrm{CP}$ phase $\phi$, we simulate, for illustration, the T2(H)K experiment, as shown in Fig. 1. The pseudo-data are simulated with the true value of $\delta_{C P}=3 \pi / 2$, under the assumption of unitary mixing.

To extract the sensitivity on the leptonic Dirac CP phase $\delta_{C P}$, we fit the pseudo-data with a $\chi^{2}$ function with current prior knowledge on the mixing angles and Eq. 1.2 in the non-unitary case. We then fit the data under different assumptions for each value of the $\mathrm{CP}$ phase $\delta_{C P}$. The blue curves in Fig. 1 are obtained by assuming standard unitary mixing. One can see that T2K can distinguish reasonably well a nonzero Dirac CP phase from zero, while T2HK can further enhance this sensitivity, under the unitarity assumption. We then turn on the non-unitarity parameters and $\chi_{\text {non-unitary. }}^{2}$ As we can see, the situation totally changes once non-unitarity is introduced. Given a nonzero fitting value $\delta_{C P}^{f i t}$, one can find a "counter-term" from the non-unitarity terms leading to agreement with the pseudo-data. In other words, the effect of the CP phase $\delta_{C P}$ can be faked by its non-unitary counterpart $\phi$. The resulting $\chi^{2}\left(\delta_{C P}\right)$ becomes nearly flat, as shown by the red curves in Fig. 1. Under the assumption of non-unitary mixing, there is almost no CP sensitivity in either $\mathrm{T} 2 \mathrm{~K}$ or T2HK. 

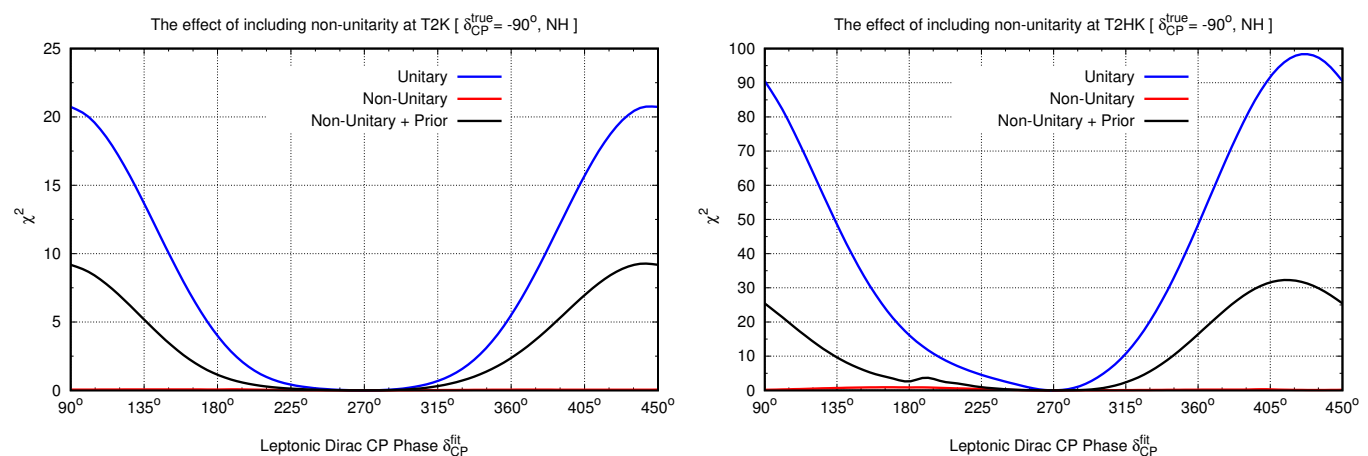

Figure 1: The marginalized $\chi^{2}\left(\delta_{C P}\right)$ function at T2K and T2HK under the assumptions of unitary mixing (blue) and non-unitary mixing with (red) or without (black) the prior constraint.

\section{Probing CP violation with $\mu \mathrm{DAR}$ and Near Detector}

In order to fully resolve the degeneracy between the unitary and non-unitary $\mathrm{CP}$ phases, it is necessary to bring back the $\cos \delta_{C P}$ dependence by carefully choosing the energy spectrum and baseline configuration. A perfect candidate for achieving this is to use muon decay at rest ( $\mu \mathrm{DAR})$ which has a wide peak and shorter baseline around $15-23 \mathrm{~km}$. The TNT2K experiment [6] is proposed to supplement the existing Super-K detector and the future Hyper-K detector with a $\mu$ DAR source. We find that the CP sensitivity is significantly improved by the combination of $\mu \mathrm{Kam}$ and
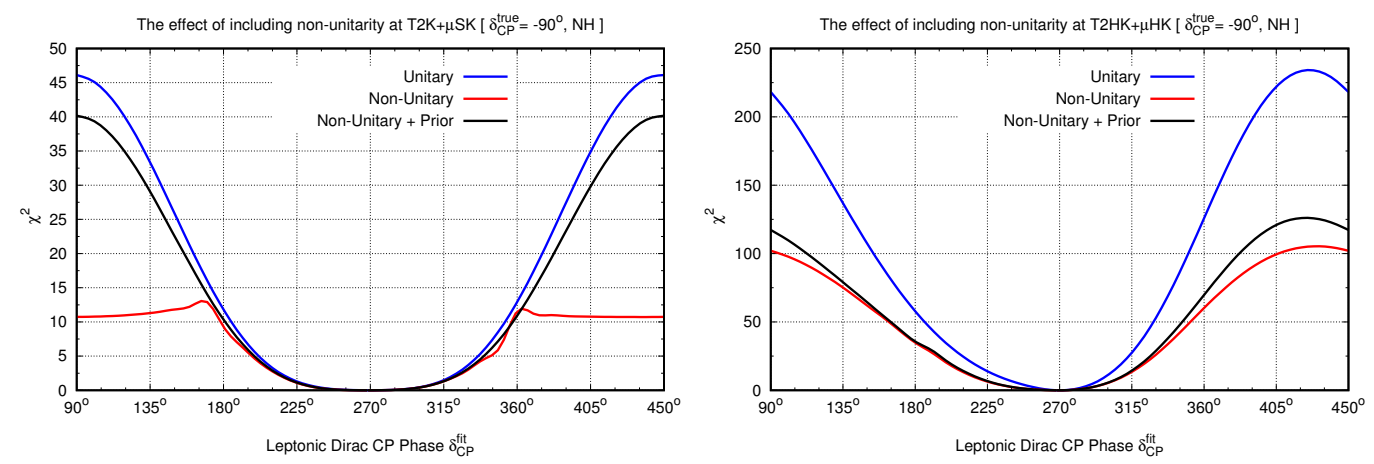

Figure 2: The marginalized $\chi^{2}\left(\delta_{C P}\right)$ function at TNT2K under the assumptions of unitarity (blue), nonunitary mixing without (red) or with (black) the prior constraint.

prior constraints. Notice in Fig. 2 that the ambiguity of the phases is partially resolved.

In the left panel of Fig. 3 we show the sensitivity on $\left|\alpha_{21}\right|$ as a function of the background rate and the detector size from a simplified template fit. In Fig. 3, right, we show the CP sensitivity at TNT2K plus $\mu$ Near once a full simulation is performed. Imposing all the information we can get from TNT2K, $\mu$ Near, the CP sensitivity can match the full potential of TNT2K under the assumption of unitary mixing.

\section{Conclusion}

Unambiguous understanding of reality always requires distinguishing alternative assumptions 

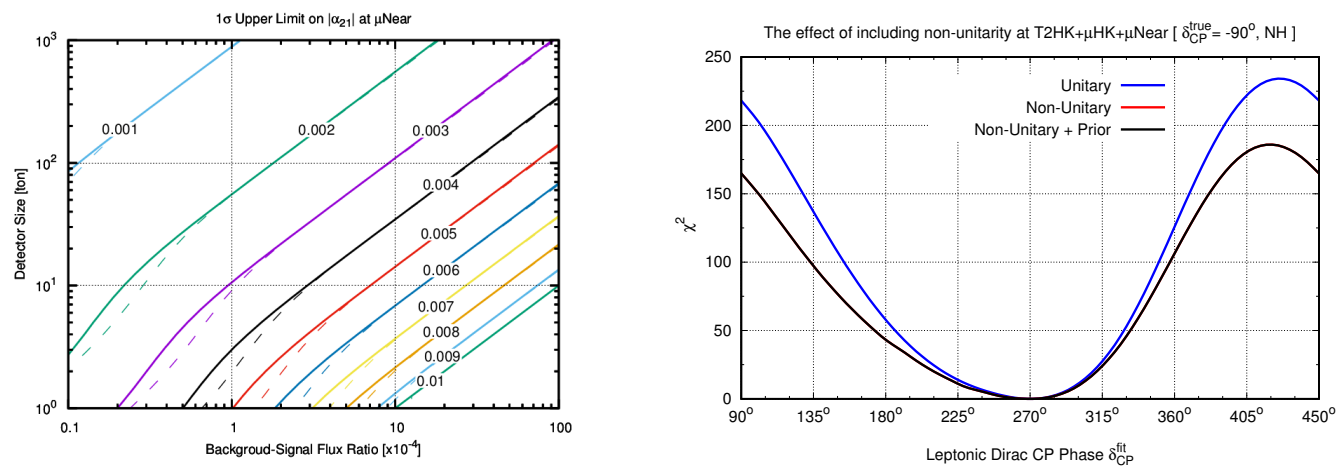

Figure 3: left: the sensitivity on $\left|\alpha_{21}\right|$ at $\mu$ Near as a function of background rate and detector size. Right: The marginalized $\chi^{2}\left(\delta_{C P}\right)$ function at TNT2K $+\mu$ Near under the assumptions of unitarity (blue), nonunitary mixing without (red) or with (black) the prior constraint.

through careful experimental design. The degeneracy between unitary and non-unitary CP phases in neutrino mixing provides a perfect example. In this paper we have confirmed, in agreement with Ref. [5], that, for values of $\left|\alpha_{21}\right|$ of the order of a few $\%$, one can have unitarity violating CP oscillation amplitudes of the same order than the standard one associated to $\delta_{C P}$. We have illustrated how the $\mathrm{CP}$ sensitivity at accelerator neutrino experiments like $\mathrm{T} 2(\mathrm{H}) \mathrm{K}$ is severely degraded in the presence of non-unitarity as in the $\mathrm{T} 2(\mathrm{H}) \mathrm{K}$ configuration. A perfect solution comes from the TNT2K project: $\mathrm{T} 2(\mathrm{H}) \mathrm{K}$ supplemented by a $\mu \mathrm{DAR}$ source. Thanks to the different energy scale of the accelerator and $\mu \mathrm{DAR}$ neutrino fluxes, two different measurements can proceed at the same time, using Super-K/Hyper-K detectors simultaneously. However, TNT2K can fully explore its advantage only in combination with a near detector. We propose using $\mu$ Near, with 20 ton of scintillator and $20 \mathrm{mS}$ baseline, to monitor the size of the non-unitary $\mathrm{CP}$ violating term for the $\mu \rightarrow e$ transition, $\left|\alpha_{21}\right|$. We have seen that the further addition of a near detector to the $\mu \mathrm{DAR}$ setup has the potential of removing the degeneracy rather well. Furhter discussion is found in [1].

\section{Acknowledgement}

Work supported by Spanish grants FPA2014-58183-P, Multidark CSD2009-00064, SEV-2014-0398 (MINECO), PROMETEOII/2014/084 (Gen. Val.). M. T. is supported by Ramón y Cajal contract (MINECO). P. S. P. thanks the support of FAPESP/Capes funding 2014/05133-1, 2015/16809-9, 2014/19164-6. Shao-Feng Ge, thanks Jarah Evslin for useful discussions.

\section{References}

[1] S. F. Ge, P. Pasquini, M. Tortola and J. W. F. Valle, arXiv:1605.01670 [hep-ph].

[2] J. Schechter and J. W. F. Valle, Phys. Rev. D 22, 2227 (1980).

[3] F. J. Escrihuela, et al., Phys. Rev. D 92, 053009 (2015).

[4] S. F. Ge, NuPro: a simulation package for neutrino properties http:// nupro. hepforge.org.

[5] O. G. Miranda, M. Tortola and J. W. F. Valle, Phys. Rev. Lett. 117, 061804 (2016).

[6] J. Evslin, S. F. Ge and K. Hagiwara, JHEP 1602, 137 (2016). 